\title{
Letter \\ Two devils: hypernatremia and hyponatremia can show faces to the same patient in the intensive care unit
}

\author{
Payam Eghtesadi-Araghi ${ }^{1}$, Mohammad R Rasouli ${ }^{2}$ and Majid Mokhtari ${ }^{3}$
}

\author{
${ }_{1}^{1}$ Parsteb Pajouheshyar Medical Sciences Research Institute, Department No. 5, 37th (Eastern), First Golzar St., Ashrafi Esfahani Blvd., Ponak Sq., \\ Tehran 1476783476, Iran \\ 2Sina Trauma and Surgery Research Center, Tehran University of Medical Sciences, Sina Hospital, Hassan-Abad Sq, Tehran, 11365-3876, Iran \\ ${ }^{3}$ Department of Medicine, Internal Medicine, Pulmonary and Critical Care, Shahid Beheshti University of Medical Sciences, Imam Hosein Public \\ Hospital, Shaheed Madani St., Tehran, 1617763141, Iran
}

Corresponding author: Payam Eghtesadi-Aragh, payam.eghtesadi@yahoo.com

Published: 6 March 2009

Critical Care 2009, 13:404 (doi:10.1186/cc7722)

This article is online at http://ccforum.com/content/13/2/404

(c) 2009 BioMed Central Ltd

See related research by Stelfox et al., http://ccforum.com/content/12/6/R162

In an article by Stelfox and colleagues published in the December 2008 issue of Critical Care, the authors tried to determine the incidence of sodium disturbance (SD) and risk factors of SD during intensive care unit (ICU) admission [1]. The impact of SD on patient mortality was also evaluated.

In this large study, the patients were divided into three groups of acquired hyponatremia, always normal sodium, and acquired hypernatremia according to their first episode of SD after arrival in the ICU. The categorization of the patients based on the first episode of SD, although simple and informative, we think oversimplifies a complex and common disorder in the ICU. Critically ill patients in the ICU may experience multiple episodes of SD, hypernatremia or hyponatremia.
A significant hypernatremic episode and its impact on final outcome could therefore easily be missed in a final analysis of patients who experienced a transient hyponatremia as the first episode of SD or vice versa. In other words, examining the impact of SD on the final outcome of patients in the ICU should take into consideration the spectrum of SD in a given patient in the ICU rather than an episode of hyponatremia or hypernatremia in isolation. It is not clear in their paper whether the investigators examined the effects of mixed SD happening in the same patient during their ICU stay.

One could therefore think that in such well-powered studies, with the potential to yield significant results, it would have been more appropriate to stratify patients into four groups: acquired hyponatremia, always normal sodium, acquired hypernatremia, and mixed SD.

\section{Authors' response \\ Henry T Stelfox, David Zygun, Kevin Laupland and Sofia B Ahmed}

Dr Eghtesadi-Araghi, Dr Rasouli and Dr Mokhtari are correct that a critically ill patient may experience multiple distinct SDs, including both hyponatraemia and hypernatraemia, during a single ICU admission and that these disturbances may influence the patient's outcome. In our population-based study of critically ill medical-surgical patients we found that $25 \%$ of the patients with a SD experienced more than one distinct SD during their ICU stay [1]. Distinct episodes of both hyponatraemia and hypernatraemia were experienced by $6 \%$ of patients with ICU-acquired SDs during their ICU stay
[1]. In examining patient outcomes, we considered serum sodium levels to be a continuous time-dependent variable, we made no assumptions about the nature, frequency or severity of SDs and we identified that both ICU-acquired hyponatraemia and hypernatraemia are associated with increased inhospital mortality in a dose-response fashion [1]. We agree that SDs in critically ill patients may be complex, that caution is prudent in interpreting the disturbances and that studies are needed to establish the optimal management strategies.

$\mathrm{ICU}=$ intensive care unit; $\mathrm{SD}=$ sodium disturbance. 
Critical Care Vol 13 No 2 Eghtesadi-Araghi et al.

\section{Competing interests}

The authors declare that they have no competing interests.

\section{Reference}

1. Stelfox HT, Ahmed SB, Khandwala F, Zygun D, Shahpori R, Laupland $\mathrm{K}$ : The epidemiology of intensive care unit-acquired hyponatremia and hypernatremia in medical-surgical intensive care units. Crit Care 2008, 12:R162. 